\title{
Multi-Branched Blood Vessels Segmentation Based on Phase-Field and Statistical Model
}

\author{
Shifeng Zhao, Mingquan Zhou, Zhongke Wu, Yun Tian, and Lizhi Xie
}

\begin{abstract}
The precise segmentation of cerebral vessels is essential for the detection of cerebrovascular diseases. The complex structures of cerebral vessels and the low contranst of thin vessels in medical images make precise segmentation difficult. In this study, we propose a new phase-field and statistical model for blood vessel segmentation. The proposed model is based on the Allen-Chan equation with double well potential and statistical distribution function. The brain tissues in the image are modeled by Gaussian distribution while cerebral vessels are modeled by uniform distribution respectively. The region distribution information combined with the phase-field model is used in curve evolution. And the level set method is developed to implement the curve evolution to assure high efficiency of the cerebrovascular segmentation. Comparisons with the LBF model and LCV model show that our model can achieve better results with fewer iteration number and less time.
\end{abstract}

Index Terms - Segmentation, cerebral blood vessel, intensity inhomogeneity, statistical model.

\section{INTRODUCTION}

Vascular segmentation is one of the fundamental tasks in the diagnosis and treatment planning of many different pathologies including arteriovenous malformations (AVMs), aneurysm, thrombosis and cardiac disease. Thus, accurate detection and segmentation of brain blood vessels is of major importance to the radiologists [1], especially those dim small ones. Therefore the accurate method of performing blood vessel segmentation on medical images of different modalities is a subject of much research attention [2].

A variety of methods have been developed for segmenting blood vessels. From the technical aspect, there is no general technique that may be effectively applied to all modalities. They have to be highly adapted to the application in order to achieve good performance. One of the general approaches for image segmentation is the minimizer of the piecewise constant Mumford-Shah functional using the variational-PDEs. Mumford-Shan function model [3] was firstly proposed as a general image segmentation model. Using this model the image is decomposed into some regions. Inside each region, the original image is approximated by a smooth function. Since the great success of curve evolution [4] and level-set method [5] in image segmentation for

Manuscript received December 5, 2012; revised February 15, 2013. This work was supported by the National Natural Science Fundation of China under Grant No.61170203, Grant No.61170170 and Grant No.61003134.

S. Zhao, M. Zhou, Z. Wu, Y. Tian, and L. Xie are with College of Information Science and Technology, Beijing Normal University, 100875, Beijing, China (e-mail:zhaosf8109@gmail.com, mqzhou@bnu.edu.cn, zwu@bnu.edu.cn, tianyun@bnu.edu.cn, Rokage@163.com).
Mumford-Shah type model, different approaches have been tried to apply such method to image segmentation [6]-[12]. For example, Chan and Vese(CV) [6] proposed a level-set framework to minimize the so-called piecewise constant model by assuming that an image consists of statistically homogeneous regions. But $\mathrm{CV}$ model has intrinsic limitations such as the unsuccessful segmentation of images with intensity inhomogeneity, the sensitivity to the placement of initial contour and the extraordinary time-consumption. So many methods have been put forward to solve the limitations of CV models [13]-[16]. Solem et al. [13] and xia et al. [14], they improved the initialization prcess, while in [15] their purpose is to reduce the computational load of the curve evolution. Wang et al. [16] introduced a local Chan-Vese model which utilized both global information and local image for segmentation. They also introduced a new penalizing energy and a new termination criterion to deal with the iteration process.

Recently, the Allen-Cahn equation [17], also known phase field model, has attracted the attention of some scholars, and it has also proved efficiency in image segmentation problems [18]-[23]. For example, Jung et al. [21] proposed a phase-field method to solve mutliphase piecewise constant segmentation problem. The method is based on the phase transition model of Modica and Mortola with a sinusoidal potential and a fitting term. It is a variational partial differential equation approach that is closely connected to the Mumford-Shah model. Chen [22] extended the sine-sinc model to Gaussian-distribution-like image. They chose a normalization of the original image as initialization of the iterations to help convergency and replace the sinc function by the exponential function to improve the efficiency of the model. Li [23] proposed a phase-field model which was based on the Allen-Cahn equation with a multiple well potential and a data-fitting term. By using the polynomial potential, they can derive a more efficient and accurate numerical scheme based on an operator splitting technique.

A major difficulty in segmentation of blood vessels is the intensity inhomogeneity. Though many methods are proposed to solve the problem of non-uniform characteristics, they are still build on the original MS model. And the fundamental nature of the MS model is piecewise smooth. So such method is still sensitive to the selection of initial curves or sensitive to noises. Meanwhile, non-uniform property is little taken into account during the process. Furthermore, most of them are tested on small size images. For larger ones, they may not be bound to get good segmenting results. Due to the disturbance of the noise and the volume effect in medical images, the intensity of different brain tissues overlap with each other. Also different imaging devices, and even the different imaging method of the same imaging device, the 
sensitivity and specificity of the different vascular are also different. So, it is unreasonable to take the original image as a piecewise constant model. As a matter of fact, in medial images, the intensity of various elements or components are also different, even the same tissue, they may take on different intensity and obey some certain distribution. The objective of this study is to propose a new method for blood vessel segmentation using phase-field and statistical model which is based on the Allen-Cahn equation with a double well potential and a statistical data fitting term.

The reminder of this paper is organized as follows: In Section II, LCV model for image segmentation is briefly reviewed. In Section III, the proposed model for image segmentation is given. Section VI is the implementation. In Section V, we exhibit experiment results for different kinds of images. We especially take a comparison our model with other two models. Finally, we close the paper with a short conclusion.

\section{DesCription OF THE PROPOSED MODEL}

\section{A. Local Chan-Vese Model}

The Local Chan-Vese(LCV) model proposed by Wang et al. [16] is composed of three terms,i.e.,global term,local term and the regularization term. And the energy functional can be described as :

$$
E^{L C V}\left(c_{1}, c_{2}, C\right)=\alpha \cdot E^{G}+\beta \cdot E^{L}+E^{R}
$$

where $E^{G}, E^{L}, E^{R}$ represent the global term, the local term and the regularization term respectively.

The global term $E^{G}$ is directly derived from the $\mathrm{CV}$ model:

$$
\begin{aligned}
E^{G}= & \int_{\text {inside }(C)}\left|f_{0}(x, y)-c_{1}\right|^{2} d x d y \\
& +\int_{\text {outside }(C)}\left|f_{0}(x, y)-c_{2}\right|^{2} d x d y
\end{aligned}
$$

where $f_{0}$ is the given image, $c_{1}$ and $c_{2}$ are the intensity averages of $f_{0}$ inside $C$ and outside $C$,respectively.

The local term $E^{L}$ using the local statistical information to improve the segmentation capability can be written as:

$$
\begin{aligned}
E^{L} & =\int_{\text {inside }(C)}\left|g_{k} * f_{0}(x, y)-f_{0}(x, y)-d_{1}\right|^{2} d x d y \\
& +\int_{\text {outside }(C)}\left|g_{k} * f_{0}(x, y)-f_{0}(x, y)-c_{2}\right|^{2} d x d y
\end{aligned}
$$

where $g_{k}$ is a averaging convolution operator with $k \times k$ window. $d_{1}$ and $d_{2}$ are the intensity average of difference image $g_{k} * f_{0}(x, y)-f_{0}(x, y)$ inside $C$ and outside $C$,respectively.

And the third term $E^{R}$ includes length penalty term $L(C)$ relating to the length of the evoluting curve $C$ and a metric term $P(\phi)$ which charactirizes how close a function $\phi$ is to a signed function:

$$
E^{R}=L(C)+P(\phi)=\mu \cdot \int_{C} d p+\int_{\Omega} \frac{1}{2}(|\nabla \phi(x, y)|-1)^{2} d x d y(4)
$$

where $\mu$ is the parameter which can control the penalization effect of the length term.

\section{B. New Model}

Because the intensity of various brain tissues is not necessarily consistent. They may be subject to different distribution. We analyzed different images and found that the histogram always has one peak near the low intensity region, which is not surprising since intensity inhomogeneity often occurs in medical images. The distribution characteristics is meaningful feature which can facilitate segmentation. So in order to get better segmentation result, we extend the model in the following steps.

First, from the above analysis, we will assume that the image consists of two classes, vessel and non-vessel. Non-vessel class includes the low intensity region and is modeled by a Gaussian distribution. The vessel class includes arteries and is modeled by a uniform distribution. The distribution of the image can be expressed as a finite mixture of two classes: vessel and non-vessel:

$$
f(x)=P\left(x \mid \omega_{1}\right) P\left(\omega_{1}\right)+P\left(x \mid \omega_{2}\right) P\left(\omega_{2}\right)
$$

where $f(x)$ is the total distribution of image pixels. $x$ is the intensity. $P\left(x \mid \omega_{1}\right)$ is the posterior distribution of non-vessel class. $P\left(x \mid \omega_{2}\right)$ is the posterior distribution of vessel class. $P\left(\omega_{1}\right)$ and $P\left(\omega_{2}\right)$ are the proportion of non-vessel and vessel in image respectively. A Gaussian distribution models non-vessel class as follows:

$$
P\left(x \mid \omega_{1}\right)=\frac{1}{\sqrt{2 \pi \sigma^{2}}} \exp \left(-\frac{(x-\mu)^{2}}{2 \sigma^{2}}\right)
$$

A uniform distribution models vessel class as follows:

$$
P\left(x \mid \omega_{2}\right)=\frac{1}{I}
$$

where, $I$ is the maximum intensity in the image.

Second, inspired by the multiphase image segmentation via Modica-Mortola phase transition, we use Allen-Chan equation to replace the length of the segmenting the curve $C$ and use the statistical distribution to replace the global term, then we will get get our phase-field and statistical model, so the energy functional can be written as:

$$
\begin{aligned}
& E(\phi)=\int_{\Omega}\left(\frac{F(\phi)}{\varepsilon^{2}}+\frac{|\nabla \phi|^{2}}{2}\right) d x d y+ \\
& {\left[\int_{\text {inside }(C)}\left|g_{k} * f_{0}(x, y)-f_{0}(x, y)-d_{1}\right|^{2} d x d y+\right.} \\
& \left.\int_{\text {ousside }(C)}\left|g_{k} * f_{0}(x, y)-f_{0}(x, y)-d_{2}\right|^{2} d x d y\right]+ \\
& \left(\frac{1}{\sqrt{2 \pi \sigma^{2}}} \exp \left(-\frac{\left(f_{0}-\mu\right)^{2}}{2 \sigma^{2}}\right) P(\phi)+\frac{1-P(\phi)}{I}\right) \sin c^{2}(\phi-k)
\end{aligned}
$$


where $F(\phi)=0.25\left(\phi^{2}-1\right)^{2}$ is a double well potential. The small constant $\varepsilon$ is the gradient energy coefficient related to the interfacial energy, $\Omega$ is the image domain, $P(\phi)$ is the proportion of non-vessel class in the image. $f_{0}$ is the given image, $\mu$ is the mean, $\sigma^{2}$ is the variance of the Gaussian distribution, $I$ is the maximum intensity in the image. And the constant functions $d_{1}, d_{2}$ are given as:

$$
\begin{aligned}
& d_{1}=\frac{\int_{\Omega}\left[g_{k} * f_{0}(x, y)-f_{0}(x, y)\right] H_{\varepsilon}(\phi(x, y)) d x d y}{\int_{\Omega} H_{\varepsilon}(\phi(x, y)) d x d y} \\
& d_{2}=\frac{\int_{\Omega}\left[g_{k} * f_{0}(x, y)-f_{0}(x, y)\right]\left[1-H_{\varepsilon}(\phi(x, y))\right] d x d y}{\int_{\Omega}\left(1-H_{\varepsilon}(\phi(x, y))\right) d x d y}
\end{aligned}
$$

Once $\phi$ comes to a steady state, the evolving curve $C$ will separates the blood vessels form the background. From this purpose, we seek a law of evolution in the form:

$$
\phi_{t}=-\operatorname{grad} E(\phi)
$$

The symbol 'grad' here denotes the gradient in the space $L^{2}(\Omega)$.then we have:

$$
\begin{gathered}
\phi_{t}=-\operatorname{gradE}(\phi)=-\frac{F^{\prime}(\phi)}{\varepsilon^{2}}+\Delta \phi \\
+\left[\left(g_{k} * f_{0}(x, y)-f_{0}(x, y)-d_{1}\right)^{2}\right. \\
\left.+\left(g_{k} * f_{0}(x, y)-f_{0}(x, y)-d_{2}\right)^{2}\right] \\
-\lambda\left[\left(\frac{1}{\sqrt{2 \pi \sigma^{2}}} \exp \left(-\frac{\left(f_{0}-\mu\right)^{2}}{2 \sigma^{2}}\right) P(\phi)+\frac{1-P(\phi)}{I}\right)\right] \\
\left(\frac{\sin (2 \pi(\phi-k))}{\pi(\phi-k)^{2}}-\frac{2 \sin ^{2}(\pi(\phi-k))}{\pi^{2}(\phi-k)^{3}}\right)
\end{gathered}
$$

where $N$ is the number of pixels of the image, $x_{i}$ is the intensity of pixel $i$.

\section{IMPLEMENTATION}

In our numerical experiments, we normalize the given image $f$ as $f_{0}=\frac{f-f_{\min }}{f_{\max }-f_{\min }}$, where $f_{\max }$ and $f_{\min }$ are the maximum and the minimum values of the given image, respectively. When the distribution is Gaussian, the parameters of the distribution can be derived from the following:

$$
P^{(n)}\left(\omega_{k} \mid x_{i}\right)=\frac{P^{(n)}\left(x_{i} \mid \omega_{k}\right) P\left(\omega_{k}^{(n)}\right)}{\sum_{j=1}^{2} P^{(n)}\left(x_{i} \mid \omega_{j}\right) P\left(\omega_{j}^{(n)}\right)}
$$

$$
\begin{aligned}
P^{(n+1)}\left(\omega_{1}\right) & =\frac{1}{N} \sum_{i=1}^{N} P^{(n)}\left(\omega_{1} \mid x_{i}\right) \\
\mu_{k}^{(n+1)} & =\frac{\sum_{i=1}^{N} P^{(n)}\left(\omega_{i} \mid x_{i}\right) x_{i}}{\sum_{i=1}^{N} P^{(n)}\left(\omega_{i} \mid x_{i}\right)} \\
\left(\sigma^{(n+1)}\right)^{2} & =\frac{\sum_{i=1}^{N} P^{(n)}\left(\omega_{1} \mid x_{i}\right)\left(x_{i}-\mu^{(n+1)}\right)^{2}}{\sum_{i=1}^{N} P^{(n)}\left(\omega_{1} \mid x_{i}\right)}
\end{aligned}
$$

\section{EXPRIMENT RESULTS}

In this section, the proposed algorithm has been tested on real blood vessels. Fig. 1 shows the segmentation results for three real blood vessel images with inhomogeneous intensity via use of the the Local Binary Fitting mode[12], Local Chan- Vese model[16] and the proposed method. In [16] they had compared the CV model with the LCV model. So we just compare LBF and LCV model with our proposed model. It can be seen from the results that the proposed model can achieve good segmenting results.

From the above experimental results, our method illustrates the ability of segmenting image with the intensity inhomogeneity and the images contain more perplexing vascular. The three images all have the characteristics of multi-branched. Because the real blood vessels in human brain take on such features as complicated and arranged in a crisscross pattern. As we all know,cerebral blood vessels are composed of a large number of branched structures, which form a highly entangled web. The methods used for blood vessel segmentation discussed in introduction can work well to deal with images of little size and the images just include one or two banch vessels. But actually, to deal with images containing more complicated branched blood vessels has more practical significance.

In real vessel images, within blood vessels, the intensity may be homogeneity, but surrongding the vicinity of vascular edges, the intensity are usually different. Because the background are normally composed of different brain tissues, they generaly have different density. So using different distribution functions to represent the different organizations is meaningful.

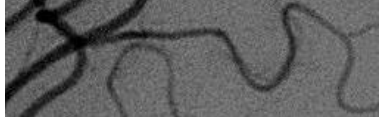

(a)

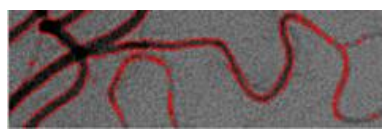

(c)

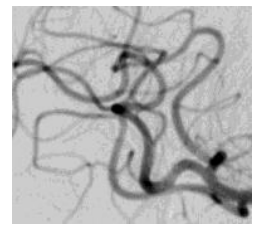

(e)

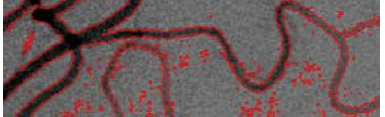

(b)

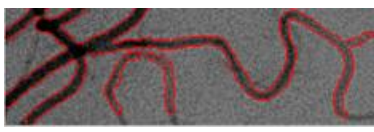

(d)

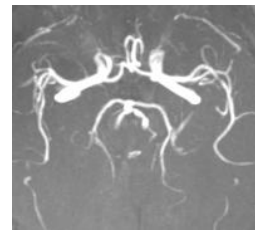

(f) 


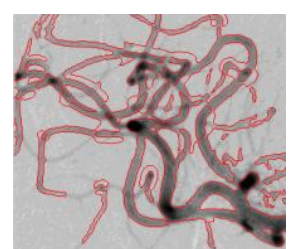

(g)

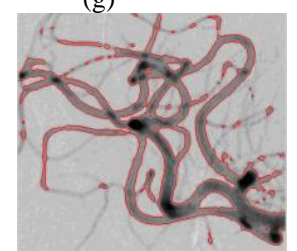

(i)

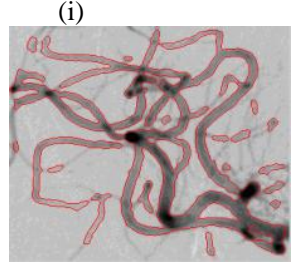

(k)

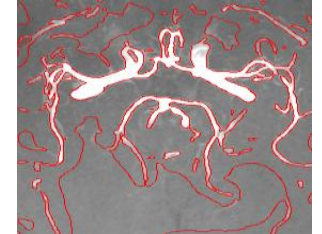

(h)

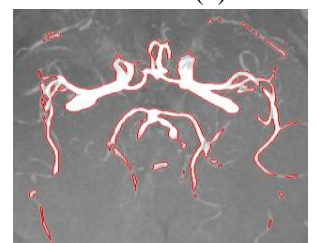

(j)

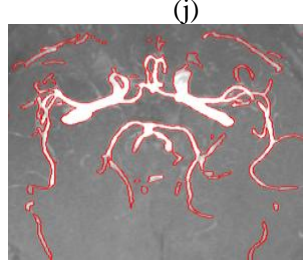

(1)
Fig. 1. The comparisons of the three models on segmenting real blood image with the intensity inhomogeneity . (a)(e)(f): original images. $(b)(g)(h)$ : final segmentation result using LBF model. (c)(i)(j): final segmentation result using LCV model. $(\mathrm{d})(\mathrm{k})(\mathrm{l})$ : final segmenting results using the proposed model.

Their iteration number and processing time for segmenting images in Fig. 1 are presented in Table I. It can be seen form the table that the iteration numbers and the processing time for the proposed model are both less than those of other two models.

For the table above, we also come to that, our proposed model not only has the ability of segmenting blood vessels with intensity inhomogeneity, but also has more advantages in handling larger images. When dealing with larger images, our method can get better results with less iteration number and less time.

TABLE I: THE PERFORMANCE COMPARISONS OF THREE MODELS

\begin{tabular}{ccccccc} 
& \multicolumn{2}{c}{ size $\mathbf{2 5 5} * \mathbf{7 7}(\mathbf{a})$} & \multicolumn{2}{c}{ size $\mathbf{3 1 9} * \mathbf{2 9 4}(\mathbf{e})$} & \multicolumn{2}{c}{ size $=\mathbf{3 1 4} * \mathbf{3 4 0}(\mathbf{f})$} \\
\hline & $\begin{array}{l}\text { Iteration } \\
\text { number }\end{array}$ & $\begin{array}{l}\text { Total } \\
\text { time }\end{array}$ & $\begin{array}{l}\text { Iteration } \\
\text { number }\end{array}$ & $\begin{array}{l}\text { Total } \\
\text { time }\end{array}$ & $\begin{array}{l}\text { Iteration } \\
\text { number }\end{array}$ & $\begin{array}{l}\text { Total } \\
\text { time }\end{array}$ \\
LBF & 20 & 16.7 & 50 & 52.2 & 100 & 88.9 \\
model & 20 & 14.4 & 20 & 22.5 & 80 & 87.6 \\
$\begin{array}{c}\text { LCV } \\
\text { model } \\
\begin{array}{c}\text { Proposed } \\
\text { model }\end{array}\end{array}$ & 10 & 9.87 & 18 & 17.1 & 60 & 55.7 \\
\hline \hline
\end{tabular}

\section{CONCLUSION}

In this study, we propose a new model for blood vessel image segmentation, which is based on the techniques of curve evolution and global statistical distribution and level set theory. The energy functional for the proposed model consists of the double well potential and a statistical data fitting term. By incorporating the global statistical distribution information into the model, the images with intensity inhomogeneity can be efficiently segmented. The experiment results show that the proposed model is better than other similar methods in medical image segmentation. In our future work we will add more image properties and make use of different geometry features to improve the segmentation results and make our method more robust.

\section{REFERENCES}

[1] S. Kostopoulos et al., "A hybrid pixel-based classification method for blood vessel segmentation and aneurysm detection on CTA," Computers \& Graphics, vol. 31, issue 3, pp. 493-500, June 2007.

[2] K. Allen, C. Yau, and J. A. Noble, "A recursive stochastic vessel segmentation framework that robustly handles bifurcations," in Proc. 12th Annu. Conf. Medical Image Understanding and Analysis, Dundee, Scotland, 2008.

[3] D. Mumford and J. Shah, "Optimal approximations of piecewise smooth functions and associated variational problems (with J. Shah)," Comm. in Pure and Appl. Math., vol. 42, pp. 577-685, 1989.

[4] L. M. Lorigo, O. D. Faugeras, W. E. L. Grimson, R. Keriven, R. Kikinis, A. Nabavi, and C.-F. Westin, "CURVES: Curve evolution for vessel segmentation, medical image analysis," vol. 5, pp. 195-206, 2001.

[5] R. Malladi, J. A. Sethian, and B. Vemuri, "Shape Modeling with Front Propagation: A Level Set Approach," IEEE Trans. on Pattern Analysis and Machine Intelligence, vol. 17, no. 2, February 1995.

[6] T. Chan and L. Vese, "Active contours without edges," IEEE Trans. Image Process, vol.10, issue 2, pp. 266-277, 2001.

[7] A. Tasi and A. Yezzi, "Curve evolution implementation of the Mumford-Shah Functional for image segmentation, denoising, interpolation and magnification," IEEE transactions on image processing, vol. 10. no. 8, pp. 1169-1186, August 2001.

[8] L. Vese and F .Tony, "A multiphase level set framework for image segmentation using the mumford and shah model," International Journal of Computer Vision, vol.50, issue 3, pp. 271-293, 2002.

[9] X. Tai and F. Chan. "A survey on multiple level set methods with applications for identifying piecewise constant functions," International Journal of Numerical Analysis and Modeling, vol. 1, issue 1, pp. 25-47, 2004.

[10] G. Chung and L. Vese, "Energy minimization based segmentation and denoising using multilayer level set approach," LNCS, vol. 3757, pp. 439-455, 2005.

[11] L. Johan, L. Marius, and X. Tai, "A variant of the level set method and applications to image segmentation," Mathematics of Computation, vol. 75, pp. 1155-1174, 2006.

[12] C. M. Li, C. Kao, J. Gore, and Z. Ding, "Implicit active contours driven by local binary fitting energy," in Proc. Conf. Computer Vision and Pattern, 2007.

[13] J. E. Solem, N. C. Overgaard, and A. Heyden, "Initialization techniques for segmentation with the Chan-Vese model," in Proc. 18th Conf. Pattern Recognition, vol. 2, pp. 171-174, 2006.

[14] R. B. Xia, W. J. Liu, J. B. Zhao, and L. Li, “An optimal initialization technique for improving the segmentation performance of Chan-Vese model, " in Proc. Confe. Automation and Logistics, pp. 411-415, 2007.

[15] Y. Pan, J. D. Birdwell, and S. M. Djouadi, "Efficient implementation of the Chan-Vese models without solving PDEs," in Proc. Workshop. Multimedia Signal Processing, pp. 350-354, 2006.

[16] X. Wang, D. Huang, and H. Xu, "An efficient local chan-vese model for image segmentation," Pattern Recognition, vol. 43, issue 3, pp. 603-618, 2010.

[17] X. Feng and A. Prohl, "Numerical analysis of the Allen-Cahn equation and approximation for mean curvature flows," Numer. Math, vol. 94 pp. 33-65, 2003.

[18] S. Esedoglu and Y. H. R. Tsai, "Threshold dynamics for the piecewise constant Mumford-Shah functional," J. Comput. Phys., vol. 211, issue 1, pp. 367-384, 2006.

[19] J. Lie, M. Lysaker, and X. C. Tai, “A binary level set model and some applications for Mumford-Shah image segmentation," IEEE Trans. Image Process., vol. 15, issue 4, pp. 1171-1181,2006.

[20] B. B. Thomas and C. Daniel, "On the statistical interpretation of the piecewise smooth mumford-shah functiona, scale space and variational methods in computer vision," Lecture Notes in Computer Science, vol. 4485/2007, pp. 203-213, 2007.

[21] Y. Jung, S. Kang and J. Shen, "Multiphase Image Segmentation via Modica-Mortola Phase transition," SIAM applied Mathematics, vol. 67, issue 5, pp. 1213-1232, 2007.

[22] F. Chen, Y. Chen and H. D. Tagare, "An extension of Sine-Sinc model based on logarithm of likelihood," in Proc. IPCV, vol. 1, pp. 222-227, 2008.

[23] Y. Li and J. Kim, "Multiphase image segmentation with a phase-field model, Computers and Mathematics with Applications," vol. 62, pp. 737-745, 2011. 


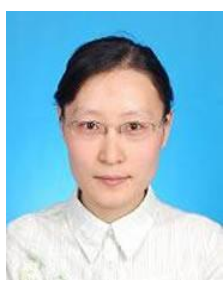

Shifeng Zhao received her B.Sc degree in Computer Science and Technology (2004) from Qufu Normal University, China. She obtained her M.Sc degree in Computer Science and Technology (2007) from Beijing Normal University, China. Since then, she has been working with the College of Information Science and Technology at BNU. Her research interests include medical imaging and virtual reality.

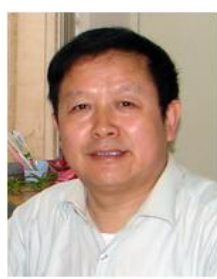

Mingquan Zhou was born in Shanxi, China in 1954. Mingquan Zhou received B. Sc in Mathematics and M. Sc in Computer Software and Theory from Northwest University, China, in 1976 and 1988 respectively. Between 1992 and 1995, he was a visiting researche at the University of Bordeaux in France. Currently, he is full Professor and $\mathrm{Ph}$. D student supervisor in the College of Information Science and Technology, Beijing Normal University (BNU), China, where he is also the dean of the College of Information Science and Technolgoy. Prof. Zhou's current research interests include computer visualization, software engineering and Chinese information processing, virtual reality and medical imaging.

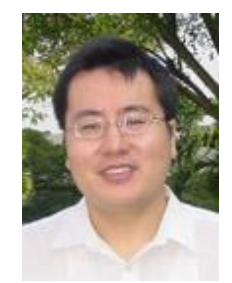

Zhongke Wu was born in Liaoning, China in 1965. Zhongke $\mathrm{Wu}$ received B.Sc in Mathematics from Peking University in China in 1988, and M. Eng and $\mathrm{PhD}$ in CAD/CAM from Beijing University of Aeronautics \& Astronautics, China, in 1991 and 1995 respectively. Currently Zhongke WU, is Full Professor and $\mathrm{Ph}$. D student supervisor in College of Information Science and Technology, Beijing Normal University (BNU), China. Prior to joining in BNU, he worked in Nanyang Technological University (NTU), Singapore, Institute National de Recherche en Informatique et en Automatique (INRIA) in France, Institute of High Performance Computing (IHPC) and Institute of Software, Chinese Academy of Sciences in China from 1995 to 2006. Prof. WU has been working in the field of computer graphics since 1988. He led and took part in various research and development projects in computer graphics and related areas. Prof. Wu's current research interests include computer graphics, animation virtual reality, geometric modeling, volume graphics and medical imaging.

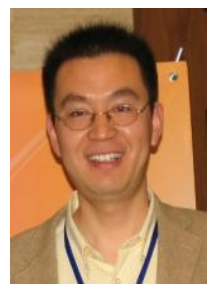

Yun Tian received his B.Sc. degree in Computer Science and Technology (2003) from Henan Normal University, China, and his $\mathrm{Ph}$. D. degree in Signal and Information Processing (2007) from Northwestern Polytechnic University, China. Currently, he is an associate professor at the College of Information Science and Technology, Beijing Normal University (BNU), China. His research interests include medical image processing and pattern recognition.

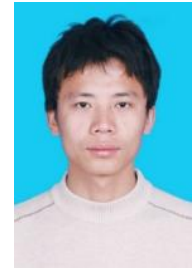

Lizhi Xie was born in 1984, doctoral level at Beijing Normal University, Beijing (BNU), China. He works in the field of medical image processing. In particular, his research interests include volume rendering of medical image, vascular segementation and vessel construction. 